\title{
Crisis Management in the Tourism Industry- The Role of Social Media Platforms
}

Jose K Antony $^{*}$ and Joel M Jacob ${ }^{\dagger}$

\begin{abstract}
The tourism industry is highly susceptible to various forms of risks operating in the industry that occur and go unattended over a period of time. Social media could be effectively used to address the risks. Studies, however, on social media and its role in crisis management are few in spite of a consistent e mphasis on the importance of social media in the tourism industry, specifically, its role in crisis management. This paper provides a review of the current state of affairs and provides a framework to involve social media research in crisis management in tourism.
\end{abstract}

Keywords: Social Media, Crisis Management, Digital Resource for Risk Minimisation

\section{Introduction to the Study}

Tourism as an industry is identified to be vulnerable to a large number of risks from various environmental factors encompassing, political, economic, socio-cultural, technological and legal, to name the most prominent ones (Faulker, 2001; Ritchie, 2004). Therefore, it is important for tourism organisations to be strategically ready and

\footnotetext{
* School of Business Studies and Social Sciences (SBSSS), CHRIST (Deemed to be University), Bangalore, India; jose.antony@christuniversity.in $\dagger$ (SBSSS), CHRIST (Deemed to be University), Bangalore, India; joelm.jacob@christuniversity.in
} 
be equipped to handle the crises that may occur due to the various risks, so as to minimise the negative impact that they could have on the industry (Blake \& Sinclair, 2003; Ritchie, 2004). Today, this is where information and communication technology (ICT) platforms and their affordances gain prominence. A major population in the ICT arena is social media platforms that include blogs, wikis, social networking sites, and media sharing sites. These platforms are increasingly being used for understanding, analysing and disseminating communication related to crises and for mobilising the support of various stakeholders to take up responsive actions and to assist in the management of the dire situations (Hughes, Palen, Sutton, Liu \& Vieweg, 2008). Twitter and other social networks coordinate and mobilise different groups of stakeholders like citizens, activists' groups, rescue teams, policy makers, police and fire forces to assist in the management of the crises.

The internet is today widely used as a tool for managing crises. The initial studies that were carried out mainly focused on the internet and its role as a medium of mass communication for supporting communications during crises (Taylor \& Perry, 2005; West, 2003; Perry, Taylor, \& Doerfel, 2003; Neil, 2000). Technologies such as interactive chats, real-time videos and audio files have also been studied as strong medium of mass communication that can help at times of crises, but the studies have not majorly focussed on the influence of the new social media platforms on interactive and peer-to-peer communication capabilities during crises (Hearit, 1999; Witmer, 2000). There have been but few studies that have analysed the impact of social media and its functions such as social networking, mass collaboration and user generated content during the management of crises (Gonzalez-Herrero \& Smith, 2008; Palen et al., 2007). These studies have been more practical in nature as they have been more descriptive and they have dealt with the actual role of social media during crisis management, which has not been tested before (Reuter, Marx, \& Pipek, 2011).

Though studies have been conducted on the significance of social media for business purposes in the tourism industry and on the management of various crises that have occurred in different parts of the world, wide gaps in research still remain. This is mainly due to the fact that studies to date have not measured the importance of 
social media and its capabilities when it comes to handling crises. The article therefore aims to address this research gap by identifying, reviewing, and consolidating all the literature related to social media and its influence on crisis management, thereby not only addressing the research implications but also the practical ramifications on the effective use of social media during a crisis.

\section{Introduction to Crisis and Crisis Management}

Of the good number of research that have introduced the concept of crisis and its management, very few have made a clear distinction between a crisis and a disaster. A crisis is normally defined as a situation which is self-made. This could be due to many reasons such as an inept management framework or due to the inability to adapt to changes. On the contrary, disasters occur when a system is suddenly confronted by a change which was highly unpredictable, which could turn out to be highly catastrophic, and over which there is very little control (Faulkner, 2001). Another study further differentiated between crisis and disaster as the unanticipated result of management failures being termed as a crisis and the unpredictable catastrophic changes after the occurrence of an event being termed as a disaster (Prideaux et al., 2003). It has to be further noted that both crisis and disaster can occur hand in hand.

As stated earlier, crisis events are highly unpredictable and they could be least expected (Coombs, 1999). Some common occurrences of crises include political unrests, terror activities, wars, natural disasters, threats due to public health, employee mistakes, erroneous decisions by the top management, industrial accidents, strikes, service failures, to name few (Sonmez, Apostolopoulos, \& Tarlow, 1999). Due to rapid globalisation and increased urbanisation, tourism entities are facing more frequent crises events. This is also further enhanced because of the over-reliance on technology and the gamut of information that all stakeholders in tourism are exposed to (Ritchie, 2004). Being an integral part of the ICT tools that are predominantly used today, social media platforms also contribute to crises as anyone can freely raise issues on these platforms which are later disseminated and discussed, sometimes in detail, thereby leading to increased perception of the 
risks involved. Hence, it is important that strategies are laid down well in order to manage such crises and ensure the necessity for the tourism organisations to survive (Ritchie, 2004; Blake \& Sinclair, 2003; Faulkner, 2001).

Crisis management can therefore be explained as the entire process of planning, confronting, responding, and overcoming a crisis. It includes all the strategies that are initiated in order to address low probability events so that the stakeholders will be able to control the management operations so as to reduce the impacts from these events (Fink, 1986). With regards to a tourism destination, crisis management usually focuses on rebuilding the destination after a crisis so that the destination will be able to recover itself after the event, thereby retaining the positive destination image and without a drop in tourist arrivals. Moreover, with the implementation of sound crisis management plans, potential aftermaths of crises can be minimised or avoided altogether (Peters \& Pikkemaat, 2005). Hence, crisis management does not always wait for the crisis to happen, but it can also mean that the destination is prepared even before the crisis strikes, so that if at all the crisis happens, the destination is ready to implement the plans and quickly recover back to the normal state within a very short period of time ( $\mathrm{Yu}$, Stafford \& Armoo, 2005).

Over the years, crisis management has emerged as a focus area of many research endeavours. Several strategic approaches and models have emerged from the studies. These studies have revealed patterns that are normally followed while handling crises and these patterns can broadly be classified under four crisis management approaches, namely the Life Cycle Approach, the Strategic Crisis Management Approach, the Action Oriented Crisis Management Approach and the Integrated Approach. All these approaches helped to better understand the crisis management processes by simplifying the complex problem domains. The Life Cycle Approach (Luhrman, 2005; Faulkner, 2001; Roberts, 1994; Fink, 1986) initially offered a three phase framework that included the pre-event, the emergency and the post-event, which was later re-modeled into six phases; the pre-event, the prodromal, the emergency, the intermediate, the long-term and the resolution stages. This re-modeling enabled to better manage the tourism 
crisis. The second approach, the Strategic Management Crisis Management Approach (Preble, 1997) consisted of three phases, the formation, the implementation and the evaluation stages. The third approach, the Action Oriented Crisis Management Approach (Wilks \& Moore, 2005; Burnett, 1998; Pearson \& Mitroff, 1993; Mitroff, 1988) described crisis management to include four stages, reduction, readiness, response and recovery (the Four $\mathrm{R}^{\prime} \mathrm{s}$ ) so as to suggest and implement various actions in order to restore tourism through effective crisis control. Finally, the Integrated Approach (Moe \& Pathranarakul, 2006) proposed both proactive and reactive strategies that enabled the stakeholders to respond to the crisis, before, during, and after the occurrence. The mitigations, preparedness and the warnings comprised the proactive strategies where as the assessments that were carried out after the events occurred comprised the reactive strategies. Both, proactive and reactive strategies are normally required to minimise chaos and social disorders during the time of crises.

\subsection{Social Media and its Use during Crisis Management}

Even though social media plays a very significant role during crises, by creating more awareness and offering a platform for wider discussions, they also have a very powerful impact as a transformational tool on the way the various stakeholders understand and respond to crises. This is mainly because social media has the ability to overcome distance and the geographical challenges during crises as the information on social media can be disseminated within less time and with far reaching view points over a wider geographical space. Social media also helps to collect and spread highly localised information, while continuously updating the same from multiple sources. This will help in decision making on how to manage the crisis during the real time situations (Sutton, Palen, \& Shlovski, 2008; Vieweg et al., 2008). Therefore, social media acts as a bridge that allows for a lot of coordination and interaction between the various stakeholders from the time of sharing of information about the crisis to the actions that are being taken to address the issue. Social media platforms also offer new ways of communication and sharing of information by always updating the information being displayed and circulated. Even the functionalities of the social media platforms offer enhanced 
visualisation capabilities and more flexible geographical interfaces that allow for better and more enhanced techniques to explore, analyse, process and then present complex information (Sigala \& Marinidis, 2012). Decision making and coordination during crises are further enhanced by the presentations of multidimensional displays of highly complex data (MacEachren \& Cai, 2006), and also through the sequential storage of large amounts of information in easily recognisable and searchable databases (Sigala \& Marinidis, 2012; Malizia, Bellucci, Diaz, Aedo, \& Leviadli, 2011). Hence, social media tools possess unique features to enhance decision making and teamwork during crises.

Research in Liberal Sciences on risk behavior also show a wide use of the Internet and other social media platforms for searching information and for using the medium for making decisions especially during tough situations (Sorensen, 2000). Many a times these platforms are used as important sources to gather information about problems so that they can be avoided from becoming crises (Thomsen, 1995; Ramsey, 1993), and this is normally achieved by increasing the proactiveness and the preparedness of the all the stakeholders involved. Social media platforms help to achieve this through collaborations and networking as they can overcome geographical distances and boundaries. The best example depicts the use of virtual communities to understand the situation better to initiate collective coordination and action to address the management of the crisis (Palen, Vieweg, Liu, \& Hughes, 2009; Palen \& Liu, 2007). Therefore, the barriers of location, the crisis condition and time zones are overcome through the effective use of social media platforms, thereby creating and fostering a more participatory culture and value system cutting across borders. All the communication that has arose through social media platforms are further stored as a collective memory (Liu, 2009) that can be used in future so as to study about how the various societies and cultures around the world used the information in order to respond and to collaborate with each other so as to manage the crises. 


\subsection{Communication using Social Media during Crisis Management}

Crisis Management deals with all the techniques that are used to address the crisis head on, and it is not just about identifying understanding and responding to the problems (Weick, 1995). Studies have dealt with the significance of communication during crisis management (Sturges, 1994). The success of crisis management can be decided by the various levels of communication that were taken up before, during and after the crisis, as studied by research (Coombs, 1999). The roles of both internal and external sources of information are also worth mentioning during the management of a crisis (Coombs, 2007; Massey, 2001; D'Aveni \& MacMillan, 1990), and the quality and the responsiveness of both these sources are an important indicator of how well prepared the organisations are in order to handle the crisis (Reilly, 2008). Again, these internal and external sources of information can contribute to the upward, downward and horizontal flow of information.

Internal communication often happens within an organisation and it normally relates to change, stress management, training, preparedness, coordination and development in crisis management, decision making and situational awareness. This form of communication helps in identifying the information and the resources that are immediately required to address the crisis situation (Lockwood, 2005). While downward communication will impose controls over the employees and their actions during the emergencies, upward communication will enable the employees in the lower levels to provide updates and suggestions to their top level managers, during the process of handling the crisis (Reilly, 1998).

External communication during crises refers to the movement of information from the organisation to all the stakeholders outside the organisation, like government bodies, activists groups and rescues forces. External communication helps to coordinate and manage the crisis by sharing not just information, but also resources. It also provides a better understanding of the crisis situation as all the stakeholders will receive a common 
understanding of the emergency due to the sharing of the correct information (Reilly, 1998).

Controlling the communication during a crisis also comes with its own challenges. There are a large number of functional, organisational and geographical boundaries to be crossed, and this is where social media can play a significant role as it allows for timely and more accurate sharing of the crisis information, across spaces and in all directions. Communication on social media platforms encompasses all stakeholders from customers to media publications. Social media platforms have also modified the way information is collected. These platforms allow for the gathering of real-time data from the crisis affected regions and people and with the help of this kind of data, up-to-date decisions can be taken for commanding relief operations and for controlling the situations (Sarter \& Woods, 1991). With more networking, collaborations and more accuracy in the information collected from across multiple sources and channels, the situational awareness can be better improved (Sonnenwald \& Pierce, 2000). And the best example for this is the use of Micro Blogging and Twitter accounts for extensive coordination between numerous crisis management groups (Vieweg, Hughes, Starbird, \& Palen, 2010).

The mobile accessibility of social media further improves the potential of these platforms to better analyse the crisis situations and the controls to be set in place. Many applications are available today that can be used in Smart Phones such as iPhones so as to offer enhanced procedures for disaster management. This is made even more significant in the present times as everyone carry their mobile phones with them during emergency situations, and this allows for easy communication with them as their location and safety can be easily verified. Also, it has to be taken into account that the applications which are used in the smart phones are highly user friendly. This has opened up the market for companies to design and manufacture such kind of applications for smart phones that work on crisis management information system, and all these interfaces work on social media platforms and participatory web mapping services (Sarcevic, Palen, \& Burd, 2011; Liu \& Palen, 2009). 


\subsection{Implications of using Social Media during Crisis Management}

Organisations in the travel and tourism industry have to be oriented and well prepared so as to effectively use social media platforms in their crisis management strategies. For which, numerous issues have to be accounted for: the type of social media platforms that have to be used at every stage of crisis management; how the various types of social media platforms have to be matched so that they can all be used efficiently in order to effectively address the crises; the technical and the design configurations of the interfaces that will be used to support the social media infrastructure; the amount, the type and the quality of information that have to be collected and used on the various social media platforms; the skills and the responsibilities of the different stakeholders when it comes to handling and processing the information on the social media platforms; and the technical knowhow, the procedures and the processes needed to monitor and manage the information about crises on social media.

Based on the crisis and the nature of the organization, that is, handling the situation, the amount of investment, and the commitment and the effort will differ in integrating the social media tools into the interface so as to manage the crisis. Studies have not really evaluated the effectiveness of each of the social media platforms that are available in order to be used during various stages of crisis management. These platforms may vary in the way they handle information, coordinate and communicate this information with the different stakeholders. Researchers are also not very clear about how the stakeholders view each of the social media platforms and which platforms they choose to adopt during different emergency situations. Studies can also be carried out from the perspectives of the tourism organisations and how comfortable they are with the use of various social media platforms during crises, while also focussing on the factors that could influence the selection of the appropriate Platforms for use during these kinds of situations.

Normally, the event and its nature decide on the most appropriate social media platform to be used. Events differ in terms of their characters, their predictabilities and the magnitude of their impacts 
because of which they are classified into small (emergencies), medium (disasters) and large (catastrophes) events. The social media that are used for each of these categories of events also differ (Quarantelli \& Dynes, 2006). The appropriateness and the effectiveness of each social media platform will vary when used for different forms of crisis events and in their different stages. Moreover, the involvement of the government and the security of the information that is used and circulated can also decide on the type of social media platforms that are used. Government agencies mainly use more safe and secure social media systems and the interoperable feature of the platforms also play an important role when it comes to sharing of the platforms and the information on it with different stakeholders.

The studies to date have mainly discussed about the effective use of social media platforms in managing crises, but discussions have not been taken up on whether social media can trigger or create crisis. this can be explored by studying in-depth about the crises situations that had occurred earlier, such as how such situations happened and then evolved, identifying the escalations and the challenges that were encountered and how social media was used as a tool to control the situations. these studies could have serious repercussions on business as tourism organisations can use the findings from such studies to better understand about such emergency situations and how to deal with similar situations if they are to repeat in future. the organisations need not have to wait till the crises escalate into disasters because of their helplessness due to their inexperience, instead by already having information on such situations from social media platforms, they are already prepared to address the crises at the grass root levels itself.

Not to forget that with the growing need and significance of having such modern and advanced technology systems in place, the information technology vendors are forced to design and develop the technology, much needed by business organisations to handle everything about crises. Moreover, as the users of social media are increasing on a daily basis, the demand to integrate the various social media interfaces available so as to ease the creation and sharing of information using a single platform is also steadily rising. Even the numbers of applications that are incorporated into 
the interfaces are important today as the users are looking forward to more numbers, faster and more efficient number of applications that will enable them to track more information with lesser clicks.

\section{Conclusion}

This article offered an introduction on the use and significance of social media during the time of crises. The interpretive research was restricted to secondary literature but it did throw light on the emerging importance of social media as a tool for crisis management. Social media has transformed the way netizens communicate with each other and during different situations, especially in times of gathering and processing large amounts of data and generating information for decision making. They have brought in more coordination and collaborations resulting in more synergised actions, which many a time can help in the management of crises from the time the crises are anticipated. This is particularly helpful for all the stakeholders involved in the tourism industry, be it the tourists, the researchers, the organizations, the government and even the local community members as they can freely share the available information with each other and work together to mitigate the challenges emerging from the crises. Due to the vulnerability of the industry to different forms of crises, it is the need of the hour to direct more number of researches in analysing and understanding how much better and more effectively can social media platforms be used for crisis management.

\section{References}

Aguirre, B., Wenger, D., Glass, T., Diaz-Murillo, M. \& Vigo, G. (1995). The social organization of search and rescue: Evidence from the Guadalajara gasoline explosion. International Journal of Mass Emergencies and Disasters, 13(1), 93-106.

Blake, A., \& Sinclair, M. T. (2003). Tourism and crisis management: US response to September 11. Annals of Tourism Research, 30(4), 813-832.

Coombs, W. T. (2007). Ongoing crisis communication: Planning, managing, and responding (2nd ed.). Thousand Oaks, CA: Sage.

D' Aveni, R. A., \& MacMillan, I. C. (1990). Crisis and the content of managerial communications: A study of the focus of attention of top managers in surviving and failing firms. Administrative Science Quarterly, 35(4), 634657. 
Farnham, S., Pedersen, E., \& Kirkpatrick, R. (2006). Observation of Katrina / Rita Groove Deployment: Addressing social and communication challenges of ephemeral groups. Proceedings of the Conference on Information Systems for Crisis Response \& Management (ISCRAM).

Faulkner, B. (2001). Towards a framework for tourism disaster management. Tourism Management, 22(2), 135-147.

Fink, S. (1986). Crisis management: Planning for the inevitable. New York: American Management Association.

Fritz, C., \& Mathewson, J. (1957). Convergence behavior in disasters: A problem in social control. Washington: Academy of Sciences, national research Council.

Gonzalez-Herrero, A., \& Smith, S. (2008). Crisis communications management on the Web: How Internet-based technologies are changing the way public relations professionals handle business crises. Journal of Contingencies and Crisis Management, 16(3), 143-153.

Hagar, C. (2006). Using research to aid the design of a crisis information management course. Presented at Association of library $\mathcal{E}$ Information Science Educators SIg: multicultural, ethnic \& humanistic concerns (MEH), information seeking and service delivery for communities in disaster/crisis. San Antonio, TX.

Hearit, K. M. (1999). Newsgroups, activist publics, and corporate apologia: The case of Intel and its Pentium chip. Public Relations Review, 25(3), 291-308.

Hughes, A., \& Palen, L. (2009). Twitter adoption and use in mass convergence and emergency events. Proceedings of the 6th International ISCRAM Conference, May 2009, Gothenburg, Sweden.

Hughes, A. L., Palen, L., Sutton, J., Liu, S. B., \& Vieweg, S. (2008). “Site-seeing” in disaster: An examination of online social convergence. In Proceedings of the 5th International ISCRAM Conference, Washington, DC.

Kendra, J. M. \& Wachtendorf, T. (2003). Reconsidering convergence and converger: legitimacy in response to the World Trade Center disaster. In lee Clarke (ed.), Terrorism and Disaster: New Threats, New Ideas. Research in Social Problems and Public Policy, 11, 97-122.

Liu, S. (2009). Informing design of next generation social media to support crisis-related grassroots heritage. PhD Colloquium of the 6th International ISCRAM Conference, Gothenburg, Sweden, May 2009.

Liu, S., \& Palen, L. (2009). Spatiotemporal mashups: A survey of current tools to inform next generation crisis support. Proceedings of the 6 th International ISCRAM Conference, Gothenburg, Sweden, May 2009.

Liu, S. B., Palen, L., Sutton, J., Hughes, A. L., \& Vieweg, S. (2008). In search of the bigger picture: The emergent role of online photo sharing in times of disaster, Proceedings of the 5th International ISCRAM Conference, Washington, DC, 140-149. 
MacEachren, A. M., \& Cai, G. (2006). Supporting group work in crisis management: Visually mediated human-GIS-human dialogue. Environmental and Planning B: Planning and Design, 33, 435-456.

Malizia, A., Bellucci, A., Diaz, P., Aedo, I., \& Levialdi, S. (2011). eStorys: A visual storyboard system supporting back-channel communication for emergencies. Journal of Visual Languages \& Computing, 22(2), 150-169.

Massey, J. E. (2001). Managing organizational legitimacy: Communication strategies for organizations in crisis. Journal of Business Communication, 38(2), 153-182.

Massey, J. E., \& Larsen, J. P. (2006). Crisis management in real time. Journal of Promotion Management, 12(3), 63-97.

Neil, B. (2000). Crisis Management and the Internet. Ivey Business Journal, 64(3), 13-17.

Palen, L., Anderson, K. M., Mark, G., Martin, J., Sicker, D., Palmer, M., \& Grunwald, D. (2010). A vision for technology-mediated support for public participation and assistance in mass emergencies and disasters. Proceedings of ACM-BCS Visions of Computer Science 2010.

Palen, L., Vieweg, S., Liu, S. B., \& Hughes, A. L. (2009). Crisis in a networked world: Features of computer-mediated communication in the April 16, 2007, Virginia Tech Event. Social Science Computer Review, 27, 467-480.

Palen, L., Vieweg, S., Sutton, J., Liu, S. B., \& Hughes, A. (2007). Crisis informatics: Studying crisis in a networked world, Third International Conference on e-Social Science, Ann Arbor, Michigan.

Quarantelli, E. L., \& Dynes, R. R. (2006). Handbook of Disaster Research. New York: Springer.

Reilly, A. H. (2008). The role of human resource development competencies in facilitating effective crisis communication. Advances in Developing Human Resources, 10(3), 331-351.

Reuter, C., Marx, A., \& Pipek, R. (2011). Social software as an infrastructure for crisis management: A case study about current practice and potential usage. Proceedings of the 8th International ISCRAM Conference, Lisbon, Portugal, May 2011.

Ritchie, B. (2004). Chaos, crises and disasters: A strategic approach to crisis management in the tourism industry. Tourism Management, 25(6), 669-683.

Sigala, M., \& Marinidis, D. (2012). Web map services in tourism: A framework exploring the organizational transformations and implications on business operations and models. International Journal of Business Information Systems, 9(4), 415-434.

Sonmez, S., Apostolopoulos, Y., \& Tarlow, P. (1999). Tourism in crisis: Managing the effects of Terrorism. Journal of Travel Research, 38(1), 1318.

Sonnenwald, D. H., \& Pierce, L. G. (2000). Information behavior in dynamic group work contexts: Interwoven situational awareness, dense social networks and contested collaboration in command and control. Information Processing and Management, 36, 461-479. 
Sorensen, J. (2000). Hazard warning systems: A review of 20 years of progress. Natural Hazards Review, 1, 119-125.

Stallings, R., \& Quarantelli, E. (1985). Emergent citizen groups and emergency management. Public Administration Review, 45, 93-100.

Starbird, K., \& Palen, L. (2011). Voluntweeters: Selforganizing by digital volunteers in times of crisis. In Proceedings of the CHI Conference 2011, May 7-12, 2011, Vancouver, BC, Canada.

Sturges, D. L. (1994). Communicating through crisis. Management Communication Quarterly, 7(3), 297-316.

Sutton, J., Palen, L., \& Shklovski, L. (2008). Back-channels on the front lines: Emerging use of social media in the 2007 Southern California wildfires. In Proceedings of the Conference on Information Systems for Crisis Response and Management (ISCRAM) 2008.

Taylor, M., \& Perry, D. (2005). Diffusion of traditional and new media tactics in crisis communication. Public Relations Review, 31, 209-217.

Thomsen, S. R. (1995). Using online databases in corporate issues management. Public Relations Review, 21, 103-122.

Tierney, K. J., Lindell, M. K., \& Perry, R. W. (2001). Facing the unexpected: Disaster preparedness and response in the United States. Washington, DC: Joseph Henry Press.

Vieweg, S., Hughes, A. L., Starbird, K., \& Palen, L. (2010). Micro blogging during two natural Hazards events: What twitter may contribute to situational awareness. Presented at the CHI Conference, April, 10-15, 2010, Atlanta, USA.

Vieweg, S., Palen, 1., Liu, S. B., Hughes, A. L., \& Sutton, J. (2008). Collective intelligence in disaster: Examination of the phenomenon in the aftermath of the 2007 Virginia Tech shootings, Proceedings of the 5th International ISCRAM Conference, Washington, DC, 44-54.

West, M. B. (2003). Crisis management's real-time frontier: Using new technology to secure critical data. Public Relations Strategist, 9(3), p. 30.

Witmer, D. F. (2000). Spinning the Web: A handbook for public relations on the internet. New York: Addison Wesley Longman.

Zook, M., Graham, M., Shelton, T., \& Gorman, S. (2010). Volunteered geographic information and crowd sourcing disaster relief: A case study of the Haitian earthquake. World Medical \& Health Policy, 2(2), 7-33. 